newborn Wistar rats, exposed to LPS at p3, were treated with rhTSG-6 i.p. (four repetitive doses of $2.25 \mathrm{mg} / \mathrm{kg}$ every $12 \mathrm{~h}$, first dose three hours before LPS-injection).

Results Investigations of TSG-6's developmental brain expression showed a linear increase from $\mathrm{p} 1$ to $\mathrm{p} 15$ on gene level. Additionally, different expression was detected in Cortex, Thalamus and Striatum on gene level at p6. Expression of TSG- 6 after LPS treatment (0-24h) was significantly increased on gene level and tendentiously on protein level. cCaspase-3, a marker of apoptosis, showed a significant down-regulation of $>30 \%$ under additional TSG-6 treatment versus sole LPS exposure ( $\mathrm{n}=12-14, \mathrm{p}=0,025)$.

Conclusions TSG-6 Expression is developmentally regulated and increased after LPS exposure. The reduction of activated Caspase-3 demonstrates the neuroprotective potential of exogenous TSG-6 administration in inflammatory-induced developmental brain injury.

\section{METHEMOGLOBIN DETERMINES DEGREE OF PRO- INFLAMMATION AND CHOROID PLEXUS APOPTOSIS FOLLOWING INTRAVENTRICULAR HEMORRHAGE IN PRETERM RABBIT PUPS}

doi:10.1136/archdischild-2012-302724.0310

S Sveinsdottir, MG Olsson, M Cinthio, M Mögelin, B Åkerström, D Ley. Lund University, Lund, Sweden

Background and Aims Inflammation following intraventricular hemorrhage (IVH) has been shown to cause periventricular brain damage. Released free hemoglobin may be an early up-stream initiator of inflammation in the intraventricular space and cause damage to the choroid plexus. We aimed to evaluate release of free hemoglobin, the relationship to inflammation in the intraventricular space and effects on choroid plexus epithelium following IVH.

Methods IVH was induced in preterm rabbit pups. Intraventricular samples of cerebrospinal fluid (CSF) were obtained under guidance of high-frequency ultrasound at 24, 48 and $72 \mathrm{~h}$ following IVH for measurement of free oxyhemoglobin $(\mathrm{OxyHb})$, methemoglobin $(\mathrm{MetHb})$ and TNFa. Analysis of gene expression (RT-PCR) for TNFa, IL-1b and heme-oxygenase-1 (HO-1) and immunostaining with electronmicroscopy (EM) was performed in choroid plexus obtained at $72 \mathrm{~h}$.

Results Intraventricular CSF concentrations of free $\mathrm{OxyHb}$ remained stable whereas those of Met $\mathrm{Hb}$ and TNFa increased from 24 to $72 \mathrm{~h}$. There was a highly positive correlation between concentrations of $\mathrm{MetHb}$ and TNFa in CSF at 72h $(\mathrm{r}=0.98, \mathrm{p}<0.0001)$. Levels of mRNA for HO-1, IL-1 $b$ and TNF-a were up-regulated in choroid plexus following IVH as compared to controls (all $p<0.01$ ). EM showed choroid plexus epithelium with fragmented microvilli, apoptotic bodies, swollen mitochondria and increased epithelial expression of TNFa following IVH (fig. 1).
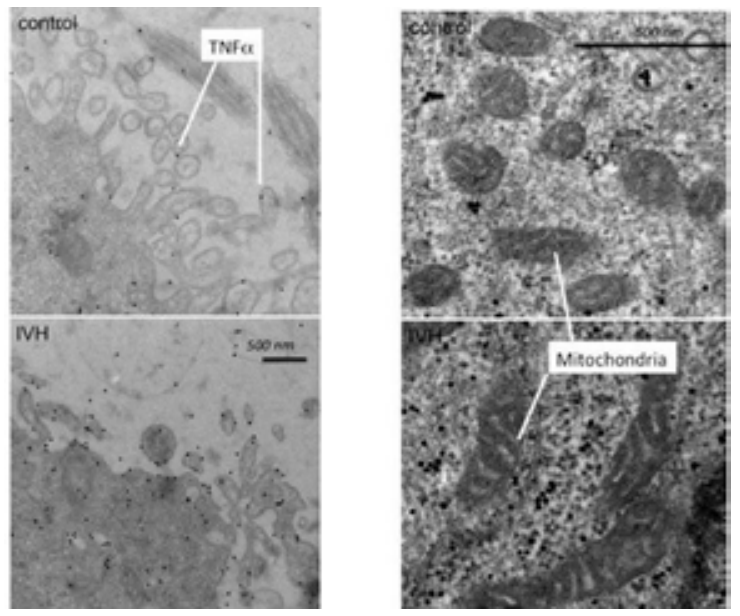

Abstract 310 Figure 1
Conclusions Amount of released free $\mathrm{Hb}$, subsequently auto-oxidised to $\mathrm{MetHb}$ determines degree of pro-inflammation in intraventricular CSF following IVH. This is associated with severe apoptosis in choroid plexus epithelium.

\section{THE SIGMA-RECEPTOR-AGONIST 4-PHENYL-1-(4- PHENYLBUTYL) PIPERIDINE (PPBP) DECREASES NEONATAL EXCITOTOXIC BRAIN INJURY AND DIMINISHES MICROGLIAL CELL ACTIVATION}

doi:10.1136/archdischild-2012-302724.0311

K Wegleiter, N Lippert, A Posod, V Neubauer, M Urbanek, U KiechlKohlendorfer, E Griesmaier. Paediatrics IV, Medical University Innsbruck, Innsbruck, Austria

The pathogenesis of perinatal brain injury is multifactorial. In animal models of adult brain injury the sigma-1-receptor agonist 4-phenyl-1-(4-phenylbutyl) piperidine (PPBP) was shown to be neuroprotective due to modulation of inflammatory and apoptotic pathways and affection of excitotoxic signaling cascades.

In the current study we evaluated the effect of PPBP in a neonatal animal model of excitotoxic brain injury.

We performed an intracranial injection of ibotenate in CD1 mice on postnatal day 5 (P5) to induce excitotoxic injury. A single injection of vehicle or PPBP, in a dosage of 1,5 or $50 \mu \mathrm{g} / \mathrm{g}$ body weight, was applied intraperitoneally one hour after the insult. Primary study endpoints were evaluated 24 (P6) and 120 (P10) hours after the insult by analysis of lesion size, number of cells stained positively for cleaved caspase- 3 and isolectin B4.

Systemic application of PPBP resulted in a significant reduction of lesion size in cortical gray matter in all dosages $(p<0.05)$. and underlying white matter in 1 and $50 \mu \mathrm{g} / \mathrm{g}(\mathrm{p}<0.05)$ at P10. At P6 microglial cell activation was significantly diminished by PPBP in cortical gray $(p<0,001)$ and underlying white matter $(p<0.05)$. Lesion size on P6 and number of cells stained positively for activated caspase-3 at P6 was not affected by the treatment ( $p>0.05$ ).

In this neonatal animal model we were able to demonstrate a beneficial effect of the sigma-1 receptor agonist PPBP. This data suggest that PPBP may be a potential therapeutic approach in neonatal brain injury.

\section{INTERMITTENT HYPOXIA: EFFECTS ON BRAIN STEM OF OXIDATIVE STRESS AND NRF2 TRANSCRIPTION FACTOR ACTIVATION IN A RAT PUP MODEL}

doi:10.1136/archdischild-2012-302724.0312

M Vento. Division of Neonatology, University and Polytechnic Hospital La Fe, Valencia, Spain

Background Apnea of prematurity is a common condition in the neonatal period caused by immature brainstem respiratory neural output. Apneic episodes and resultant intermittent hypoxia are a potential cause of oxidative stress during this vulnerable developmental period.

Objective We tested the hypothesis that chronic intermittent hypoxia alters oxidative metabolism and resultant redox status in the medulla of rat pups.

Design/methods Litters of 10 rat pups and their dams were assigned to one of two groups: normoxia (controls) and intermittent hypoxia $(\mathrm{Hx})$. Exposure occurred from postnatal day 1 for 7 days. Chronic intermittent hypoxia consisted of exposing rat pups and their dams to alternating cycles of nitrogen $\left(\mathrm{N}_{2}\right)$ and room air (RA): 45 seconds of hypoxia (nadir of $5 \% \mathrm{O}_{2}$ ) was administered every 5 minutes for 8 hours/day. For controls, animals were kept at RA. On the eighth day, brainstems were harvested and quickly snap frozen in liquid nitrogen. Reduced (GSH) and oxidized (GSSG) glutathione, and the GSH precursors -glutamyl-cysteine (-G-cysteine) 
and L-cysteine in medulla were determined by UPLC-MS/MS Malondialdehyde (MDA) in medulla was determined by HPLC.

Results Reduced glutathione (GSH) was significantly reduced in the brainstem of rat pups submitted to chronic intermittent hypoxic episodes associated with reduction in GSH/GSSG ratio. GSH precursors, -glutamyl-cysteine and L-cysteine were also significantly lower in the brainstem of intermittent hypoxia group.

\section{INTERACTION OF INFLAMMATION AND HYPEROXIA IN NEONATAL WHITE MATTER DAMAGE}

doi:10.1136/archdischild-2012-302724.0313

${ }^{1} \mathrm{~F}$ Brehmer, ${ }^{2} \mid \mathrm{Bendix},{ }^{3.4 Y}$ van de Looij, ${ }^{5} \mathrm{M}$ Sifringer, ${ }^{3} \mathrm{~S}$ Sizonenko, ${ }^{6} \mathrm{C}$ Mallard, ${ }^{1} \mathrm{C}$ Bührer, ${ }^{2} U$ FelderhoffMüser, 'B Gerstner. 'Neonatology, Charité Universitätsmedizin Berlin, Berlin; 2Department of Pediatrics 1, Neonatology, University Hospital Essen, Essen, Germany; ${ }^{3}$ Department of Pediatrics, University of Geneva, Geneva: ${ }^{4}$ Laboratory of Functional and Metabolic Imaging, Ecole Polytechnique Fédérale de Lausanne, Lausanne, Switzerland; ${ }^{5}$ Department of Anesthesiology and Intensive Care Medicine, Charité Universitätsmedizin Berlin, Berlin, Germany; ${ }^{6}$ Physiology, University of Gothenburg, Gothenburg, Sweden; 'Paediatric Heart Center, University Hospital Giessen, Giessen, Germany

Intrauterine infection/inflammation are major causes of preterm birth. The dramatic rise of oxygen tissue tension compared to intrauterine conditions amounts to relative hyperoxia in preterm infants. Both, infection/inflammation and hyperoxia have been shown to be involved in brain injury of preterm infants. Hypothesizing an additive or synergistic effect, we investigated the influence of a systemic lipopolysaccharide (LPS) application on hyperoxia-induced white matter damage (WMD) in newborn rats.

Three-day-old Wistar rat pups received $0.25 \mathrm{mg} / \mathrm{kg}$ LPS i.p. and were subjected to $80 \%$ oxygen on P6 for $24 \mathrm{hrs}$. WMD was assessed by immunohistochemistry, western blot and diffusion tensor magnetic resonance imaging. In addition, LPS and hyperoxia were studied in an in vitro co-culture system of primary rat oligodendrocytes and microglia cells. Both noxious stimuli, hyperoxia and LPS, induced a significant increase in apoptotic cell death as revealed by elevatation of cleaved caspase-3 and TUNEL-positive cells. Furthermore, both hyperoxia and LPS caused hypomyelination, as revealed by western blot, immunohistochemistry and altered WM microstructure on MRI. However, the combination of hyperoxia and LPS did neither increase nor decrease cell death and hypomyelination in vivo. In contrast, LPS pre-incubation reduced oligodendrocyte susceptibility towards hyperoxia in vitro.

Our data suggest that inflammation and hyperoxia strongly attenuate oligodendrocyte maturation by apoptotic and nonapoptotic pathways. If both insults are combined, second phase releases of protective cytokines can partially prevent oligodendrocyte cell death. The knowledge of interactions between inflammation and hyperoxia might offer new therapeutic opportunities to prevent $\mathrm{WMD}$ in preterm infants.

\section{HYPEROXIA CHANGES THE BALANCE OF THE THIOREDOXIN/PEROXIREDOXIN SYSTEM IN THE NEONATAL RAT BRAIN}

doi:10.1136/archdischild-2012-302724.0314

${ }^{1} \mathrm{~K}$ Strasser, ${ }^{1} \mathrm{I}$ Bendix, ${ }^{2} \mathrm{U}$ Weichelt, ${ }^{2} \mathrm{~S}$ Endesfelder, ${ }^{3} \mathrm{C}$ von Haefen, ${ }^{4} \mathrm{R}$ Heumann, ${ }^{4} \mathrm{~A}$ Ehrkamp, ${ }^{2} \mathrm{C}$ Buehrer, 'U Felderhoff-Mueser, ${ }^{2,3} \mathrm{M}$ Sifringer. 'Department of Pediatrics 1. Neonatology, University Children's Hospital, Essen; ${ }^{2}$ Department of Neonatology; ${ }^{3}$ Department of Anaesthesiology and Intensive Care Medicine, Charite Campus Virchow, Berlin; ${ }^{4}$ Department for Molecular Neurobiochemistry, Ruhr-University Bochum, Bochum, Germany

Background and Aim As demonstrated previously, oxygen contributes to the pathogenesis of neonatal brain damage and leading to neurocognitive impairment of prematurely born infants in later life.
Reactive oxygen species (ROS) and intrinsic antioxidant defense systems play an important role in both physiological cell signaling processes and many pathological conditions, including neurodegenerative disorders and oxygen-toxicity. Beside the glutathione-system several other redox-modulating proteins are known to be involved in redox-homeostases. The aim of this study was to evaluate potential alterations within the thioredoxin/peroxiredoxin system after exposures to nonphysiologic high oxygen levels in the developing rat brain.

Methods Six-days old Wistar rats were exposed to $80 \%$ oxygen for $6,12,24$ or 48 hours and littermates kept in room air served as controls $(n=6-8)$. Brains (excluding cerebellum) were evaluated after perfusion with PBS and dissection of both hemispheres for RNA and protein analyses.

Results We demonstrate that elevated oxygen concentrations change the balance of the ROS-dependent thioredoxin/peroxiredoxin system. Oxygen-toxicity significantly induced upregulation of peroxiredoxins in infant rat brain. In parallel, hyperoxia reduced the level of DJ-1, a hydroperoxide-responsive protein.

Discussion These findings are highly relevant from a clinical aspect because oxygen administration to neonates is often inevitable, and we recommend that every effort should be made in neonatal medicine to limit exposure of these immature babies to high oxygen concentrations. These results may also contribute to receive optimal therapeutical approaches to ameliorate oxygen toxicity.

\section{AORTIC AND CAROTID INTIMA-MEDIA THICKENING IN APPROPRIATE-FOR-GESTATIONAL AGE PRETERM NEWBORNS WITH ADEOUATE BUT LOW BIRTH WEIGHT}

doi:10.1136/archdischild-2012-302724.0315

U Schubert. Neonatology, Karolinska University Hospital, Karolinska Institutet, Stockholm, Sweden

Purpose Assessment of intima-media thickness (IMT) of the aorta and carotid artery in appropriate for gestational age (AGA) infants during the first 6 months of life after very preterm birth.

Methods Longitudinal ultrasound assessment including 21 very preterm and 29 infants born at term (all AGA) during a six-months period corresponding to the third trimester of pregnancy and the first 3 months after term, measuring aortic and carotid IMT by an angle-corrected M-Mode, and assessment of blood pressure at final follow-up.

Results No differences in aortic or carotid IMT or blood pressure measurements were found between the two groups. However, in relation to vessel lumen diameter, IMT is significantly higher in both arteries in infants born preterm $(p=0.003$ for aorta and $p=0.001$ for carotid artery).

Conclusion In relation to vessel diameter, infants born preterm show thickening of the intima-media in the great arteries. It remains to be established whether this relative intima-media thickening persists into childhood and may be a risk marker for future cardiovascular disease among subjects born preterm.

\section{EARLY VERSUS DELAYED CYCLOSPORINE TREATMENT IN CARDIAC RECOVERY AND INTESTINAL INJURY DURING RESUSCITATION OF ASPHYXIATED NEWBORN PIGLETS}

doi:10.1136/archdischild-2012-302724.0316

${ }^{1}$ RS Gill, ${ }^{2 T F}$ Lee, ${ }^{3} \mathrm{C}$ Sergi, ${ }^{2} \mathrm{C}$ Joynt, 'DL Bigam, ${ }^{1,2} \mathrm{PY}$ Cheung. ' Surgery; ${ }^{2}$ Pediatrics; ${ }^{3}$ Pathology and Labaratory Medicine, University of Alberta, Edmonton, AB, Canada

Background Recently, we have demonstrated that treating asphyxiated newborn piglets with intravenous cyclosporine immediately following resuscitation can improve cardiac function. However, immediate treatment may not be feasible for a large portion of newborns delivered in peripheral/rural hospitals. Therefore, our 